\title{
MOVIMIENTOS SOCIALES Y EL DERECHO INTERNACIONAL: AVANCES Y RETOS*
}

\section{SOCIAL MOVEMENTS AND INTERNATIONAL LAW: PROGRESS AND CHALLENGES}

\author{
José Germán Burgos-Silva** \\ JUAN DAVID SÁNCHEZ-VARGAS***
}

\section{Resumen}

Objetivo. El siguiente escrito se propone reunir un conjunto de entradas teóricas del derecho internacional alternativas a las entradas dominantes centradas en la participación de los Estados y organizaciones internacionales en la construcción de normas internacionales. Metodología. Con base en el análisis documental se examina la clave en que se ha desarrollado la relación entre el derecho internacional y los movimientos sociales. Resultado. Como resultado se obtuvo un marco general para entender en qué términos se ha explorado dicha relación. Conclusión. Se concluye con los avances que se han gestado en esta relación, y con los retos mayores para la academia con miras a la construcción de un marco teórico del derecho internacional de los movimientos sociales.

Palabras clave: derecho internacional, derecho internacional desde abajo, movimientos sociales, perspectivas críticas del derecho internacional.

\begin{abstract}
Objective. The purpose of this article is to gather a set of theoretical entries of international law alternative to the dominant entries, which are focused only on the participation of States and international organizations in the building of international standards. Methodology. Based on a documentary analysis, the pattern in which the relationship between international law and social movements has taken place is examined. Results. As a result, a general framework to understand in which terms that relationship has been explored was obtained. Conclusions. It concludes with the advances that have been developed in this relationship, and with the major challenges for the academy in order to construct a theoretical framework of the international law of social movements.
\end{abstract}

Key words: international law, international law from below, social movements, critical perspectives of international law.

\footnotetext{
* Este artículo de reflexión es fruto de intereses comunes de los autores, se deriva de un proceso de investigación y de una discusión entre los co-autores a partir de una investigación sobre el tema elaborada por Juan David SánchezVargas y asesorada por el profesor José Germán Burgos Silva.

${ }^{* *}$ Universidad Nacional de Colombia. Bogotá, Colombia. E-mail: jgburgoss@unal.edu.co -

(D) orcid.org/0000-0001-9361-990X. Google Scholar

**** Universidad Nacional de Colombia. Bogotá, Colombia. E-mail: judsanchezva@unal.edu.co -

(D) orcid.org/0000-0002-6096-7470. Google Scholar
} 
Movimientos sociales y el derecho internacional: avances y retos

\section{Introducción}

Que los movimientos sociales han pasado en las últimas décadas a ser un actor relevante en las relaciones internacionales es una afirmación que no muchas escuelas internacionalistas pondrían en duda. Sin embargo, a la hora de hablar de movimientos sociales en el derecho internacional las palabras se quedan cortas y tal fenómeno parece hoy desconocido. Aunque no del todo, existen algunos autores que con un lente más grueso y una perspectiva crítica han dado pasos hacia el "derecho internacional de los movimientos sociales”. En este artículo nos proponemos hacer una revisión de tales perspectivas; es la reunión de manifiestos que reniegan del estadocentrismo a la hora de hablar de derecho internacional y que reclaman más atención para los actores que, valiéndose del derecho -no solo interno sino también internacional-, se resisten y libran sus propias luchas.

Boaventura de Sousa Santos es uno de los primeros autores en abordar estas nuevas dinámicas ya que abrió la puerta para que otros autores e investigadores arrojaran luz sobre este tema. Según De Sousa (1998), la entrada estratégica de movimientos sociales en luchas transnacionales en torno al derecho no se puede entender al margen de los procesos de globalización. Dichos procesos han replanteado al derecho y han conllevado la proliferación de normas e instituciones jurídicas que traspasan la escala nacional, escala que había sido la base de todo derecho moderno. Plantea entonces que las sociedades modernas están ahora inscritas en una pluralidad de ordenamientos jurídicos, por lo cual, desde un foco sociojurídico, son constelaciones jurídicas. Este fenómeno abarca a los movimientos sociales del Norte y Sur globales que encuentran en normas internacionales, fundamentalmente las concernientes al respeto por los derechos humanos, un instrumento que fortalece y hace eco de sus luchas sociales a nivel local. Este postulado sería la base analítica con la que nuevos estudios profundizaron en la relación entre derecho internacional y movimientos sociales.

En orden a lo anterior, en primera medida ahondaremos más en la propuesta de De Sousa, por lo que expondremos los principales avances que este autor dio en la materia, lo que servirá para demarcar un mapa de ruta teórico. En seguida, revisaremos otros estudios y teorías que han desarrollado más el fenómeno y que merecen ser estudiados por los elementos que aportan a la discusión y que consideramos importantes para entender mejor las perspectivas críticas del derecho internacional. Finalmente, presentaremos las pistas que quedan abiertas y que merecen ser acotadas a modo de conclusión. 


\section{Discusión}

\section{Derecho internacional y movimientos sociales}

Como más arriba lo especificamos, De Sousa fue el autor que lanzó esta discusión al centro de la palestra. Para hacer una anotación en el caso latinoamericano, De Sousa, ya en 1998, narraba - escuetamente- el fenómeno de producción de nuevos instrumentos que servían a las luchas para el caso particular de los pueblos indígenas en la arena internacional. Sobre el Convenio 169 de la OIT y, en ese entonces, el proyecto de la Declaración Universal de los Derechos de los Pueblos Indígenas de las Naciones Unidas, De Sousa expresaba que "la reivindicación de derechos colectivos por parte de los pueblos indígenas y las minorías étnicas está ganando fuerza en el programa político internacional, respaldada por una 'coalición transnacional amplia y creciente" (De Sousa, 1998, p. 160). Esta última referencia de De Sousa se entiende como la unión de ONG indígenas y no indígenas, que han estado llamando la atención al mundo sobre la violación de los derechos de los pueblos indígenas, y presionando a los Estados para detener tal situación (De Sousa, 1998).

Una década más tarde, De Sousa y Rodríguez (2007) desarrollaron más profundamente lo que la dinámica de defensa internacional de los derechos humanos ha representado para los movimientos sociales. Dicha defensa tiene como correlato lo que De Sousa denomina un sistema internacional de derechos humanos, un horizonte político esencialmente cosmopolita defendido por el conjunto de movimientos sociales alrededor del globo que reivindican la justicia social. En esta lucha el marco jurídico internacional es increpado por los movimientos sociales y es puesto en contradicción con sus propios principios procedimentales y sustantivos.

Ejemplos de esto son para De Sousa y Rodríguez (2007), la demanda y conquista de la reconstrucción multicultural de los derechos humanos hecha por los pueblos indígenas, que ha contrarrestado el prejuicio individualista liberal, connatural al nacimiento de los derechos humanos; los movimientos populares y las organizaciones que han puesto en entredicho la tradición estadocéntrica en la construcción y aplicación de instrumentos jurídicos sobre derechos humanos; asimismo, los movimientos feministas también han criticado eficazmente el núcleo patriarcal de los derechos humanos y han impulsado nuevos conceptos atravesados por la justicia de género; de igual forma, desde abajo se continúa rechazando la jerarquización de los derechos, es decir, la separación de derechos entre generaciones, y más bien se apela por articular los derechos políticos y civiles con los derechos sociales (De Sousa y Rodríguez, 2007). En razón a estas muestras subversivas, los autores consideran que las presiones de los movimientos sociales ayudan a entender lo que es la reconfiguración de los derechos humanos en clave de justicia de género, étnica, racial y económica (De Sousa y Rodríguez, 2007). 
En un marco de análisis similar, es menester indicar que el autor que quizás ha construido un entramado teórico más acabado sobre derecho internacional crítico es Rajagopal (2005). En El Derecho Internacional desde abajo. El desarrollo, los movimientos sociales y la resistencia del Tercer Mundo, este autor indio explota las herramientas de las ciencias sociales - especialmente la sociología jurídica y la sociología política- para describir y analizar el potencial y las limitaciones del uso del derecho internacional por parte de los movimientos sociales. En tal sentido, la obra de este autor tiene dos ejes básicos: las instituciones internacionales y la retórica de los derechos humanos, en ellos cuestiona cómo estos derechos se han configurado como un elemento de resistencia del Tercer Mundo. Así, sus planteamientos se ubican en las antípodas de las perspectivas dominantes sobre el derecho internacional, las cuales giran en torno a paradigmas específicos de la racionalidad y modernidad occidentales que predeterminan cuáles son los actores para los que existe el derecho internacional, principalmente, los Estados.

De este modo, adopta una perspectiva "desde abajo" del derecho internacional, lo que significa concentrarse en "la experiencia vivida de la gente ordinaria con el derecho internacional, en su trato con las instituciones internacionales, cuando tienen que presentar sus peticiones en términos jurídicos internacionales o cuando construyen redes para influir en la política internacional o doméstica” (Rajagopal, 2005, p. 21). Esta forma de entender el derecho, lleva a la idea fundamental de que el derecho internacional no es solo la materialización, por lo general convencional, de la voluntad de los Estados, sino también un instrumento que es susceptible de llenarse de nuevos contenidos; los movimientos sociales han iniciado esta tarea en recientes lustros.

Partiendo de esta premisa básica, Rajagopal (2007) realizó un estudio de caso en el cual expone las dinámicas en la India del movimiento Narmada Bachao Andolan (NBA, en español "Salvamos el Narmada”) en el uso estratégico del derecho nacional e internacional. Este movimiento surgió en 1989 como contraposición al programa del Banco Mundial para el desarrollo del valle del Narmada, que implicaba el desplazamiento de la población para la construcción de una serie de represas a lo largo del río Narmada. Luego de un proceso de resistencia en tribunales nacionales e internacionales, el NBA logró archivar el programa del Banco Mundial y conquistó la implementación de la World Commission on Dams, una política pública mucho más acorde a lo que el movimiento deseaba para su territorio (Rajagopal, 2007).

En un sentido semejante, se encuentra también la perspectiva crítica que brinda Massoud (2006), este profesor estadounidense también apuesta por la descentralización del derecho internacional del Estado y por "exponerlo en el diario vivir de las personas y los movimientos sociales" (p. 6). Por ello cuestiona en primera medida la forma en que se enseñan los derechos humanos en las facultades de derecho. Fundamentalmente porque se parte del paradigma de derecho internacional como algo propio del nivel internacional, desdeñando los avances que se han dado en el campo de los derechos humanos desde lo local. 
De lo anterior se desprende que hay una crítica adicional de Massoud que, al menos, Rajagopal no parece vislumbrar en su crítica al derecho internacional. Esta crítica descansa en la misma pedagogía del derecho, la cual peca por un exacerbado abstraccionismo del carácter internacional, al dejar de lado la enseñanza de la construcción y reformulación del derecho en los planos locales. Esto nos conduce a pensar que la raíz del desconocimiento que persiste en la relación entre los movimientos sociales y el derecho internacional está en el desinterés que se inculca en las aulas de clase a la hora de ver efectos prácticos del derecho internacional, más allá de las sentencias de tribunales internacionales.

Al apartarse de este foco y, más bien, prestar atención a la utilización de la sociedad civil del derecho internacional, Massoud (2006) encuentra las organizaciones sociales en los Estados Unidos al servicio de pequeñas comunidades que, con presupuestos pequeños, han incorporado terminología y estrategias propias del derecho internacional de los derechos humanos para reforzar sus investigaciones y sus discursos con un proyecto de globalidad de los derechos humanos.

En contravía de un primer juicio que, erróneamente, pueda considerar a este enfoque como uno desligado de la academia, Massoud (2006) también arguye que tales acciones tienen también un alcance teórico, este es, refutar el realismo en el derecho internacional y las relaciones internacionales.

\footnotetext{
Esto significa, que usando el derecho internacional exitosamente para cambiar la sociedad en sus niveles más básicos -local, individual y un cambio personal- estas organizaciones representan un reto que no ha sido respondido por la visión realista del derecho internacional. (Massoud, 2006, p. 4)
}

De esta manera, la apropiación del derecho internacional por parte de actores que podríamos denominar como subalternos, por utilizar el concepto gramsciano, pone en seria duda el foco estadocentrista que ha imperado en la enseñanza de las relaciones internacionales y, simultáneamente, cuestiona la idea del derecho internacional como instrumento al servicio de los Estados más poderosos.

Vale la pena considerar ahora que, aunque estas críticas son recientes, tienen antecedentes teóricos que datan de final del siglo pasado, por ejemplo Massoud (2006) retoma los aportes de McCann (1994), quien dedicó gran parte de su obra académica al estudio de la influencia del derecho en individuos y grupos para la construcción de movimientos sociales. El aporte de este autor es cardinal por haber mostrado que los derechos se convierten en símbolos legales que crean lazos de solidaridad y, también, que las tácticas legales pueden ser funcionales al cambio económico y político. Este planteamiento, aunque se dé de forma tácita, es transversal 
Movimientos sociales y el derecho internacional: avances y retos

a todos los autores que encuentran en el derecho un espacio de resistencia. En el caso particular de Massoud, la pretensión es llevar los planteamientos de McCann al plano internacional, siendo su tesis que los movimientos sociales en Estados Unidos han desplegado el concepto de derechos humanos internacional para "avergonzar" al Gobierno. Esto significa para McCann (Como se citó en Massoud, 2006), que el:
(...) derecho internacional de los derechos humanos es mucho más que un campo de doctrina enseñada por las facultades de derecho o creada por Naciones Unidas; o sea, las relaciones entre Estados y el grado de su cooperación. Los derechos humanos, son una rama del derecho internacional, que ha influenciado significativamente los movimientos sociales y la movilización legal. (p. 6)

Como muestra de los argumentos anteriores, Massoud (2006) estudia algunos casos de movimientos sociales que han introducido el derecho internacional, particularmente el de los derechos humanos, en sus discursos y formas de acción. Entre estos casos están los de Battered Mother's Testimony Project (Proyecto Testimonios de Madres Maltratadas) y Justice Now (Justicia Ahora), organizaciones no gubernamentales que propugnan por el respeto a los derechos humanos de las mujeres y madres, mediante la denuncia de abusos, la pedagogía y el acompañamiento psicológico. Estas estrategias siempre son formuladas en clave de los derechos humanos; de esta forma, se desarrollan informes que tienen como eje instrumentos declarativos o convencionales como la Declaración Universal de Derechos Humanos, el Pacto Internacional de Derechos Civiles y Políticos, la Convención contra la Tortura y otros Tratos o Penas Crueles Inhumanos o Degradantes o la Declaración sobre la Eliminación de la Violencia contra la Mujer (Massoud, 2006).

Además de esto, en su estudio Massoud va más allá y caracteriza la metodología de estos movimientos, lo que el autor denomina "la metodología de los derechos humanos". Dicha metodología consta, en primer lugar, de reunir información y cifras de abuso, tomando como fuente datos de organizaciones internacionales u ONG con mayor capital informacional. En segundo lugar, viene la identificación del actor gubernamental responsable de prevenir tales violaciones de derechos. En tercer lugar, está la interpretación de las violaciones de derechos humanos a la luz del cuerpo de normas internacionales sobre derechos humanos; este paso es desarrollado básicamente por abogados, quienes al analizar las entrevistas a las víctimas vinculan los hechos con tratados internacionales de protección de derechos humanos. Luego de esto, viene una tarea de publicidad de los hechos y los responsables del mismo a través de informes completos o comunicados de prensa. Finalmente, se inicia la labor de presionar al Gobierno mediante el envío de cartas y la invitación a líderes mundiales a pronunciarse sobre la situación de derechos humanos. 
Luego de este aporte - el cual sirve para identificar cuál ha sido la lógica adoptada por ciertos movimientos sociales en el despliegue del derecho internacional estratégicamente--, este autor finalmente llega a la conclusión de que son cuatro los elementos que trae el despliegue del "poder local" del derecho internacional por los movimientos sociales, a saber:

1. Proporciona una metodología para evaluar casos de abuso.

2. Legitima y universaliza la retórica de la responsabilidad internacional.

3. Influye en la propia autoestima de las víctimas.

4. Moviliza a los sujetos para conformar grupos que afirman derechos.

Planteamientos estos de relevancia por cuanto brindan elementos concretos para caracterizar en qué términos se da la relación entre los movimientos sociales y el derecho internacional.

Es importante aclarar que hay críticas bien sustentadas sobre las consecuencias nocivas que conlleva este activismo legal nutrido de los derechos humanos. Por ejemplo, la abogada y profesora checa Bukovská (2008) defiende la tesis según la cual la presentación de informes, las actividades de promoción y defensa de los derechos y el litigio estratégico, pueden ser perjudiciales y contraproducentes "para lograr el cambio deseado, porque en lugar de eliminar las relaciones de poder y de dominación sobre los que tienen por objeto beneficiar, a menudo las perpetúan” (Bukovská, 2008, p. 7,8). La argumentación de esta afirmación tiene tres pilares. En primer lugar, las víctimas, puesto que los informes tienden a reforzar el estereotipo de las víctimas como débiles, sumisas, compasivas o derrotadas. En segundo lugar, la recolección de la información sobre los casos, porque en muchas ocasiones esta información es tratada con una ética profesional cuestionable. Por último, habría un quiebre en la autonomía de las víctimas cuando un ente ajeno se apersona de sus casos, muchas veces con intereses ajenos a la causa.

No obstante, hay que hacer la salvedad de que las críticas de Bukovská se dirigen especialmente a las ONG de carácter internacional y no a los movimientos sociales o a las organizaciones de anclaje local como las que hemos tratado. Como ilustración, la autora expresa lo siguiente sobre los informes de estas organizaciones de carácter internacional:

\footnotetext{
son preparados por personas ajenas al lugar por lo tanto, deben necesariamente interpretar el lenguaje de las víctimas. A éstas (sic) no se les permite ser los sujetos activos en la producción de sus propias descripciones, pero son fuente primaria de los informes. (Bukovská, 2008, p. 11)
}

Esta crítica pone de relieve el accionar de ciertas ONG que, con el ánimo de mejorar su perfil público internacional, muestran el ángulo más dramático de la situación de las víctimas 
de quienes han tomado la vocería. Al mismo tiempo, queremos destacar que la posición de Bukovská (2008) no riñe con los enfoques que hemos venido abordando porque vela por un cambio en el activismo legal en el que se le devuelva la centralidad de la resistencia y la agencia política a las víctimas. Precisamente, cuando hablamos de perspectivas críticas del derecho internacional nos referimos a que los actores de resistencia sean las propias víctimas, quienes desde abajo adelanten sus acciones de defensa al utilizar el derecho internacional como herramienta. Hecha esta acotación, conviene continuar con los aportes feministas.

Kouvo y Pearson (2011) han compilado un conjunto de aproximaciones sobre la relación del derecho internacional con las perspectivas feministas en Feminist perspectives on contemporary international law. Between resistance and compliance? En contraste con el estudio de Massoud, este acervo de artículos pretende sembrar fundamentos no solo investigativos sino también teóricos. En razón a esto, el argumento fundamental es que la relación entre feminismo(s) y derecho internacional se mueve en dos ejes: entre la sumisión y la resistencia. Respecto del primero, las feministas estudian el cómo y por qué las mujeres han sido marginalizadas del derecho internacional. En el segundo eje, los movimientos feministas encuentran en el derecho internacional una esperanza por su potencial reivindicativo. Este último punto es en el que nos queremos detener en este escrito.

La tesis principal que defienden las autoras es que, aunque sea cierto que luego del cambio de siglo era ya claro que el feminismo, así como otras perspectivas alternativas, habían incursionado en el derecho internacional, dada la invitación de la escuela dominante occidental y liberal del derecho internacional a participar en los debates legales, políticos y para la elaboración de políticas, las lógicas del nuevo debate han estado limitadas. Ellas expresan lo siguiente sobre la irrupción del feminismo en el derecho internacional:
(...) es evidente con la aparente integración del concepto de género y problemas como los derechos de la mujer en la práctica legal internacional, así como con la ostensible aceptación de escuelas feministas legalistas en la disciplina. Sin embargo, esta "invitación" fue condicionada: a las feministas se les pidió que fueran la voz de todas las mujeres, pero no podían retar los fundamentos del derecho internacional y sus instituciones. (Kouvo y Pearson, 2011, p. 5)

Demarcado el plano de antinomia, el feminismo en el derecho internacional según Kouvo y Pearson, quienes retoman a Charlesworth (2011), necesita en primer lugar deconstruir los valores implícitos y explícitos del derecho internacional sobre el género. En otras palabras, develar los puntos ciegos que tiene el sistema internacional legal que devienen en la exclusión de las mujeres y sus experiencias. En segundo lugar, el feminismo necesita reconstruir un derecho internacional que no signifique la opresión del hombre sobre la mujer. 
No obstante, las autoras son claras en que el feminismo no puede proponerse destruir al derecho internacional - como algunas feministas postcoloniales han sugerido-, porque sería destruir también la potencialidad que ya contiene para las mujeres.

En este punto es importante anotar dos cosas respecto del intento de las autoras: primero, que el aporte de las autoras es excepcional, si tomamos en cuenta la ausencia de perspectivas feministas que se preocupen por el derecho internacional. En segundo lugar, hay que precisar que la tensión no es poca dentro de los feminismos, sin embargo, nos interesa rescatar la racionalidad feminista que se destaca por ver en el derecho internacional una oportunidad de resistencia.

Alejándonos ahora de esta perspectiva feminista, existe otro estudio que, aunque tiene un foco más amplio, es igualmente crítico. Tsutsui, Whitlinger y Lim (2012), en su texto International Human Rights Law and Social Movements: States' Resistance and Civil Society's Insistence, trazan claramente la contraposición entre el Estado y la sociedad civil en el campo del derecho internacional de los derechos humanos. Los autores establecen que luego de la Segunda Guerra Mundial:

el derecho internacional de los derechos humanos emergió como un acuerdo entre actores de la sociedad civil, que se movilizaron colectivamente para poner los derechos humanos como el principio guía en la comunidad internacional; y los Estados, la mayoría de los cuales se resisten a las presiones reformistas de la sociedad civil. (Tsutsui et al., 2012, p. 368)

Empero, tal acuerdo, aunque en un principio favorecía a los Estados, creó un marco de oportunidad lo suficientemente amplio para que los movimientos sociales disputaran el pulso para continuar avanzando en la lógica de los derechos humanos. Este pulso, según los autores, parece caer gradualmente del lado de los movimientos sociales si nos fijamos en hechos como el fortalecimiento de instrumentos normativos sobre derechos humanos y su eficacia progresiva.

Con esto, Tsutsui et al. (2012) al igual que los autores que ya hemos revisado, no quieren decir que los Estados no sigan siendo los actores más poderosos en el escenario internacional, es ostensible que no es así, es evidente que las violaciones a los derechos humanos continúan, e incluso están lejos de cesar. No obstante, lo que merece ser resaltado es que, pese a esto, los avances que se han dado en materia de derechos humanos no son desdeñables y esto tiene su raíz en el rol incansable que han jugado los movimientos sociales en la puja por ampliar el espectro de derechos y profundizar los ya reconocidos. 
Una contribución importante de este estudio descansa en una revisión histórica del nacimiento de instrumentos convencionales de derechos humanos. Si bien, todos los estudios que hemos revisado se preguntan por la actual relación entre derecho internacional y movimientos sociales, la verdad es que su relación viene de atrás y, para ser más claros, los movimientos sociales de mitad de siglo fueron los que gestaron el derecho internacional de los derechos humanos hoy vigente.

Tsutsui et al. (2012) plantean de este modo que los movimientos sociales no solo son receptores de los instrumentos sobre derechos humanos creados por los Estados, sino además que los movimientos sociales también han participado históricamente en el global lawmaking, desde el momento fundacional de los derechos humanos en el derecho internacional luego de 1945. Muestra de lo anterior, es la participación de no pocas ONG y movimientos sociales en la construcción de la Carta de las Naciones Unidas, los cuales lucharon por introducir en el preámbulo del documento el compromiso de la ONU por reafirmar la fe en los derechos humanos y promover y fortalecer el respeto a los derechos humanos y las libertades fundamentales sin distinción de raza, sexo, lengua o religión (Naciones Unidas, 1945).

Hasta aquí hemos revisado diferentes aportes y aproximaciones por lo que recapitular antes de proseguir se hace necesario. Como insumo básico existe un grupo de estudios que se funda en caracterizar al derecho internacional como una arena de lucha en la que confluyen diversos actores que tienen márgenes de acción diferentes entre sí y que van desde los Estados, pasando por las organizaciones internacionales, hasta los movimientos sociales. Partiendo de este sustento, se pueden adicionar tres elementos. En primer lugar, desde la segunda mitad del siglo pasado ha habido un auge en lo que a normativa internacional sobre derechos humanos concierne, y que constituye al día de hoy una cornucopia que nutre las reivindicaciones de los movimientos sociales. En segundo lugar, a pesar de lo anterior, el derecho internacional contiene una matriz de dominación inherente desde múltiples esferas, a saber: centroperiferia, racial, cultural y de género. Sin embargo, el derecho internacional es susceptible de ser reformulado por los movimientos sociales generando así un marco de resistencia. En tercer lugar, los actores sociales no son solo receptores del derecho internacional, por el contrario, han participado históricamente - mal que bien- en la construcción de normatividad internacional sobre los derechos humanos.

Hecha esta síntesis también hay lugar para una aclaración. En relación a los tres elementos explicados en el parágrafo anterior, hay que decir que no es menos cierto que los derechos humanos han encarnado decepciones, por una parte, porque se han usado para legitimar intereses particulares de algunos gobiernos; por otra parte, porque se han convertido en un discurso que no adquiere materialidad. Estas dos decepciones son categorizadas por Kennedy (2012) como pragmatismo e idolatría. Consideramos que el riesgo de la idolatría es especialmente importante para los movimientos sociales. De acuerdo con este 
autor, la idolatría a los derechos humanos es caer en el absolutismo ético, lo que pasa por “dejarse llevar por la promesa de los Derechos Humanos y perder de vista otras virtudes" (Kennedy, 2012, p. 24), es decir, mantener fe ciega a los derechos humanos. La dificultad radica, según Kennedy, en que la estrecha lógica de los derechos humanos dificulta reclamar como derechos, problemas colectivos como la pobreza. Del mismo modo, en la defensa de los derechos humanos se estarían defendiendo a la vez un modelo único de justicia, de relacionamiento con el Estado, y del centro y la periferia. Los siguientes casos latinoamericanos que estudiaremos podrían sustentar cómo los movimientos sociales solventan de algún modo las preocupaciones de Kennedy, al hacer una relectura de los derechos humanos.

En razón a esto, para poner el foco ahora en América Latina, hay tres estudios que vale la pena revisar. El primero de ellos realizado por Delamata (2013), politóloga y socióloga argentina, desentraña el activismo constitucional de los movimientos sociales de su país. Esta autora se esfuerza por observar la utilización de los derechos y las leyes, fundamentalmente internas, pero también internacionales, en actores sociales que tienen por fin el reconocimiento legal de sus pretensiones lo que tendría, desde el punto de vista de Delamata, efectos positivos para la democracia, procedimental y sustantivamente, al ampliar la gramática de la ciudadanía.

Delamata extiende el espectro de actores en los que cabe la apropiación del derecho como herramienta reivindicatoria, no solo ya a los movimientos sociales, sino que también, bajo la categoría de comunidad legal garantista, incluye a abogados-académicos, magistrados y jueces, como fichas de importancia a la hora del reconocimiento legal, a la hora de los usos contrahegemónicos y contestatarios del derecho, y que de la mano de movimientos sociales han construido una nueva gramática de la ciudadanía y de la democracia.

En orden a desarrollar la anterior idea, Delamata parte de dos referentes: de la mirada desde abajo del derecho, Boaventura de Sousa Santos y César Rodríguez Garavito -revisada más arriba-, y de Sieder, Schjolden y Angel. Estos últimos realizan una interesante diferenciación en los procesos de movilización legal porque argumentan que tales procesos pueden venir desde arriba, mediante actores de élite como las cortes constitucionales; desde abajo, por parte de actores sociales y, desde el extranjero, a través del activismo en tribunales internacionales o de terceros países (Delamata, 2013). Aunque no está explicitado por los autores, podemos establecer que tales formas de movilización son susceptibles de ser combinadas, por lo que no es demente plantear una movilización desde abajo y desde y hacia el extranjero simultáneamente.

En el caso que estudia la autora se descubre cómo el activismo constitucional contemporáneo en Argentina devino en la judicialización de derechos sociales en las villas de Buenos Aires. Sobre estas villas, que son barrios fuertemente afectados por la pobreza, la desigualdad social y la delincuencia, Delamata argumenta que ha habido una relectura de los derechos reconocidos localmente a la luz del derecho internacional de los derechos humanos. 
Como resultado, en las villas han ocurrido dos cosas: un aprendizaje de derechos y la mayor judicialización de sus problemáticas sociales. Esto ha impulsado a su vez dinámicas como la autoorganización de los vecinos frente a asuntos que los afectan como las elecciones representativas o los nuevos proyectos legislativos. Como consecuencia, se ha fortalecido "el repertorio de los actores populares y puede redundar, eventualmente, en una reorientación más integra de la política pública” (Delamata, 2013, p. 163).

Respecto de los migrantes, existe una aproximación a la relación entre derecho internacional y los movimientos de migrantes que nos gustaría revisar por la novedad que representa. Medina (2011) desarrolla una perspectiva desde abajo del derecho para el análisis del traslado entre sistemas de derecho. En razón a ello, la autora parte de que la situación de no-ciudadanía que tienen los migrantes, refugiados, migrantes legales o ilegales, conlleva a estas poblaciones a enfrentar necesidades diferentes y urgentes de asistencia legal, empleo o vivienda, dependiendo de la relación que se establezca con el Estado receptor. Según la autora, esta relación siempre es ambigua, pues, aunque los migrantes no están afuera del Estado, tampoco hacen parte de él (Medina, 2011). En estos factores se halla la razón para que los migrantes en busca de una ciudadanía mínima creen derecho.

El argumento básico para defender lo anterior, radica en el concepto de transnacionalismo desde abajo, desarrollado por Medina (2011). Según la autora:

\begin{abstract}
(...) los migrantes, como movimiento, son creadores de derecho, con la transnacionalización del campo jurídico y el desplazamiento de las concepciones legales nacionales, que promueven a través de la relación que ellos establecen entre las sociedades partícipes del proceso migratorio. Los migrantes dan lugar a que los sistemas de derecho de las sociedades de origen y de destino rebasen el marco legal circunscrito al Estado-nación, e involucren formas jurídicas legales e ilegales que, además de crear vías de satisfacción de sus necesidades, impregnen un carácter emancipatorio al derecho. (p. 71)
\end{abstract}

La primera parte de esta cita contiene elementos que ya hemos referenciado acá, como el descentramiento del derecho de los Estados y el tránsito hacia sociedades que son constelaciones jurídicas, en este caso particular, los movimientos de migrantes. Empero, al final hay un elemento del todo nuevo, Medina es la única autora que atañe un valor emancipador al derecho. Los autores que hemos revisado, reconocen un doble carácter del derecho internacional: un aspecto que es funcional a la dominación y un aspecto que brinda oportunidades de resistencia. Aunque De Sousa (1998) hable de un potencial emancipatorio del derecho, según este autor debe haber primero una construcción nueva del sentido común del propio derecho, lo que no aparece en el esbozo de Medina (2011). Esto lleva a pensar que el derecho actual es per se emancipatorio para la autora. 
Esto último, abre el espacio para discusiones profundas de filosofía jurídica y política en las que no nos interesa entrar ahora. Sin embargo, sí existen algunos cuestionamientos que nos gustaría plantear, por ejemplo, la autora habla de Colombianos en el Exterior, el proyecto auspiciado por empresarios para ayudar a mantener el contacto familiar de migrantes colombianos y otros proyectos en demás países para garantizar derechos mínimos. Al respecto quedan serias incertidumbres sobre cómo piensa la emancipación la autora, si como el aplanamiento de relaciones sociales, justicia distributiva o el reconocimiento cultural; o, como la simple garantía de necesidades básicas de los migrantes. Nuestra consideración es que otorgar un carácter emancipatorio al derecho de tajo, significa ignorar la dimensión negativa del mismo, y sugerir equivocadamente que el marco normativo brinda oportunidades suficientes y previas a su transformación.

Finalmente, hay un estudio de caso en Colombia sobre lo que Aguilar, Cruz, Sánchez y Torres (2016), categorizan como instrumentalización subalterna del derecho internacional. Este análisis da cuenta de la nueva racionalidad de los movimientos sociales en la arena internacional, para lo cual describen el proceso de resistencia y lucha del pueblo indígena Awá de Nariño y Putumayo, frente a frecuentes y gravísimas violaciones de derechos humanos a causa del conflicto armado interno. Los Awás han adoptado una racionalidad que se destaca por no estar anclada únicamente a las lógicas nacionales, sino que más bien su proceso de resistencia se afinca en el plano internacional. En este, el derecho internacional ha sido uno de sus principales recursos ya que han encontrado en la Comisión Interamericana de Derechos Humanos un espacio de conquista para la defensa de sus derechos.

Para Aguilar et al. (2016) esta instrumentalización subalterna del derecho internacional se construye desde actores sociales subalternos que buscan la formalización de sus reivindicaciones y las consecuentes repercusiones en su plano local. Esta investigación retoma aportes de la bidimensionalidad gruesa del derecho de la que nos habla Rajagopal (2007), a saber: "el carácter limitante que tiene el derecho frente a los movimientos sociales como dispositivo funcional al mantenimiento del status quo y, por otra parte, el margen de maniobra que brinda a los movimientos sociales elementos para la resistencia” (p. 168). Este margen, según Aguilar et al. (2016), se amplía en la medida en que aparecen factores como la multiescalaridad del derecho (local, nacional, global) que significa el crecimiento de espacios jurídicos y políticos en donde se pueden desafiar las decisiones judiciales nacionales para la defensa de los derechos.

Finalmente, una conclusión que deja este estudio radica en que, aunque el derecho internacional contiene una carga occidental ineludible, alrededor de todo el sur global múltiples organizaciones sociales con culturas no occidentales realizan una relectura permanente de los derechos humanos y con base en ella reivindican sus derechos. Esto quiere decir que los procesos de resistencia legal, en el campo nacional e internacional, no solo pasan por la utilización del derecho, sino también por la apropiación del mismo que, en últimas, deviene en 
formulaciones alternativas de los derechos de las comunidades. Por esto, es común que cada grupo étnico o pueblo indígena reivindique sus derechos en clave de la discursiva retórica de los derechos humanos con base en una interpretación propia de los mismos.

\section{Conclusiones}

Según hemos visto, no son pocos los estudios y perspectivas que han abordado la relación entre derecho internacional y movimientos sociales. No obstante, la construcción de un área de estudio sólida para esta relación parece aún distante porque, aunque abundan los estudios de caso, su base teórica es todavía difusa. La razón de esto radica en que la academia aún está por detrás de un fenómeno ostensible como la resistencia de los movimientos sociales con base en los instrumentos del derecho internacional. Por esta razón, es necesario avanzar en una sinergia académica que no relegue la potencialidad del derecho en general, y del derecho internacional en particular, ni tampoco las nuevas tendencias de los movimientos sociales con alcances transnacionales. Los autores que hemos revisado, aunque hacen un aporte importante - dado el desconocimiento y/o desinterés de este fenómeno en las facultades de derecho, ciencias políticas y ciencias humanas--, son un garbanzo de a libra, y aún es mucho lo que queda por profundizar en las perspectivas desde abajo del derecho internacional.

Por ahora, se han dado pasos nada desdeñables en el estudio de la relación, puesto que fue evidente cómo cada estudio explorado contribuyó a su modo a la discusión. Sin embargo, los adelantos se han dado de forma disgregada, es decir, aunque se han desarrollado interesantes estudios de caso que ilustran las nuevas dinámicas de los movimientos sociales con el derecho internacional, no ha habido un estudio ni multidisciplinar ni multidimensional que se aventure a dar una explicación de esta dinámica de forma concreta. Más bien, se ha avanzado primordialmente estudiando a los actores por separado, es decir, a los pueblos indígenas, a las mujeres, a los inmigrantes e incluso a los movimientos cívicos. Esto por supuesto es valioso, sobre todo si se tiene en cuenta que estas discusiones han contribuido a la descentración del derecho internacional de las lógicas estadocéntricas, poniendo al derecho internacional como un elemento que se mueve no solo de arriba hacia abajo, sino también de abajo hacia arriba. El derecho internacional corresponde a la escala internacional y global, claro, pero desde lo local el derecho internacional también es apropiado e interpretado. El mérito de esos estudios es visibilizar esa dinámica.

En orden a lo anterior, es menester dar el salto hacia la construcción teórica de un marco más amplio -aunque no general-, que brinde puntos de partida claros para comprender las dinámicas que sostienen los movimientos sociales con el derecho internacional en el contexto de globalización, con miras a superar los marcos ad hoc que se han desarrollado hasta el momento y que hemos revisado aquí. En este propósito, la clave interdisciplinar es 
deseable para avanzar en esta construcción, particularmente el diálogo entre disciplinas como el derecho, la sociología y la ciencia política, para concebir un cuerpo teórico nutrido de múltiples perspectivas que permita entender esta tendencia en los movimientos sociales.

\section{Referencias}

Aguilar, D., Cruz, J., Sánchez, J. yTorres, S. (2016). Nuevas formas de resistencia transnacional: la lucha Awá, 2009-2014. International Law, Revista Colombiana de Derecho Internacional, 29, 9-56. http://dx.doi.org/10.11144/ Javeriana.il 14-29.nfrt

Bukovská, B. (2008). Perpetrando el bien: las consecuencias no deseadas en la defensa de los derechos humanos. Sur, Revista Internacional de Derechos Humanos, 9, 6-21.

Charlesworth, H. (2011). Talking to ourselves? Feminist Scholarship in International Law. En S. Kuovo y Z. Pearson (Eds.), Feminist perspectives on contemporary international law. Between resistance and compliance? (pp. 17-32). Oregon: Hart Publishing,

Delamata, G. (2013). Movimientos sociales, activismo constitucional y narrativa democrática en la Argentina contemporánea. Sociologías, 15 (32), 148-180.

De Sousa Santos, B. (1998). La globalización del derecho. Los nuevos caminos de la regulación y emancipación. Bogotá, Colombia: ILSA - Instituto Latinoamericano de Servicios Sociales Alternativos-, Universidad Nacional de Colombia.

De Sousa Santos, B. y Rodríguez, C. (Eds.). (2007). El derecho y la globalización desde abajo. Hacia una legalidad cosmopolita. Barcelona, España: Anthropos Editorial.

Kennedy, D. (2012). The international human rights regime: still part of the problem. En R. Dickinson, E. Katselli, C. Murray y O. W. Pedersen (Eds.), Examining critical Perspectives on Human Rights perspectives on human rights (pp. 2-34). Cambridge, Inglaterra: Cambridge University Press.

Kouvo, S. y Pearson, S. (2011). Feminist perspectives on contemporary international law. Between resistance and compliance? Oregon: Hart Publishing.

Massoud, M. F. (2006). The influence of International Law on Social Movements. Peace \& Change, 31 (1), 3-34.

Medina, A. (2011). Repensar los derechos de los migrantes desde abajo. Una aproximación a la relación entre migración y derechos a partir del traslado entre sistemas de derecho. Bogotá, Colombia: Universidad del Rosario. Publishing.

Naciones Unidas. (1945). Carta de las Naciones Unidas. 1 UNTS XVI. Recuperado de http: / / www.un.org/es/charter-united-nations/index.html 
Rajagopal, B. (2005). El Derecho Internacional desde abajo. El desarrollo, los movimientos sociales y la resistencia del Tercer Mundo. Bogotá, Colombia: ILSA-Instituto Latinoamericano de Servicios Sociales Alternativos.

Rajagopal, B. (2007). Los límites del derecho en la globalización contrahegemónica: la Corte Suprema de la India y la lucha en el valle de Narmada. En B. de Sousa y C. Rodríguez (Eds.), El derecho y la globalización desde abajo. Hacia una legalidad cosmopolita (pp. 167-194). Cuajimalpa, México: Anthropos.

Tsutsui, K., Whitlinger, C. y Lim, A. (2012). International Human Rights Law and Social Movements: States' Resistance and Civil Society's Insistence. Annual Review of Law and Social Science, 8, 367-396. 